\title{
A rare case of Herpes Simplex Virus type 2 radiculo- myelitis in a young new mother
}

\author{
Cellina M*, Rosti C, Pirovano M, Ciocca M and Oliva G \\ ASST Fatebenefratelli Sacco, Ospedale. Fatebenefratelli e Oftalmico, Piazza Principessa Clotilde 3, 20121 Milano, Italy
}

\section{Introduction}

Non-traumatic myelitis is a neurological disease affecting the spinal cord that can result from a variety of pathologic conditions including infectious, parainfectious, paraneoplastic, inflammatory or metabolic disorders or ischemic alterations [1]. Infectious myelitis most frequently affects the thoracic spinal cord [2].

The most common symptoms are fever, paraparesis, variable motor, sensory, and/or autonomic dysfunction that can develop over hours to days and worsen over days to weeks [3].

Sensory symptoms usually present as ascending paresthesias, loss of sensation, with or without back pain at or near the level of the myelitis. Motor symptoms often include weakness or flaccid paresis of leg and arm muscles that preferentially interests the flexors of the legs and the extensors of the arms and can include sphincter dysfunction. Autonomic involvement manifests with urinary retention, constipation, temperature dysregulation or bouts of hypertension [4-7].

HSV 2 primary infection or reactivation within root ganglia can spread through the spinal cord causing ascending necrotizing myelitis [8-11] and, by direct extension, can involve the brainstem and lead to death, when not diagnosed and treated on time [7]. We present a case of infectious transverse myelitis secondary to HSV-2 reactivation in a young new mother patient with a presentation of paraparesis; she manifested an episode of herpes genitalis 20 days before the delivery.

\section{Case report}

We report the case of a previously healthy 37-year-old female patient, who developed, the day after delivery, urinary retention and right leg paresis, associated with lumbar pain. She was afebrile, tachycardic, with $110 \mathrm{bpm}$ and tachypneic, with $25 \mathrm{bpm}$. At the neurologic evaluation, mental state and cranial nerves were normal; she presented a hyposthenia of mild degree of the right quadriceps femoris muscle (Medical Research Council [MRC] 4) and of high degree of the right biceps femoris muscle (MRC 3), with bilateral tactile hypoesthesia, with level at the distal third of tibia on the right side and below malleolar on the left side.

Deep tendon reflexes were absent in the lower limbs and plantar responses were both extensor. In the upper extremity's strength was normal but reflexes were brisk. 20 days before the delivery she manifested a herpes genitals infection that was treated with topic therapy (Acyclovir for 3 days). The course of pregnancy and delivery had been normal.

Unenhanced lumbar MRI (Figure 1) was performed and showed segmental increase in T2 and STIR signal from conus medullaris

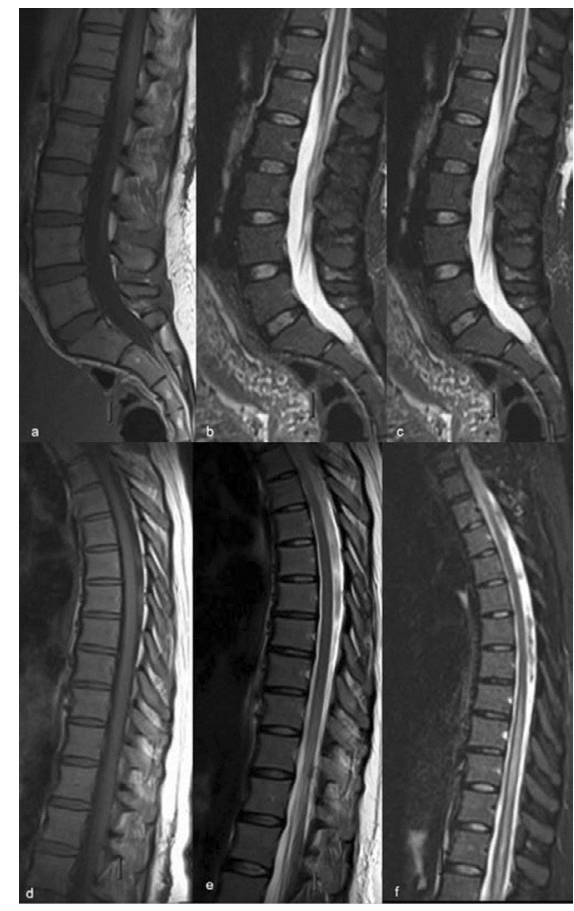

Figure 1. First unenhanced dorsal lumbar spine MRI

(a) T1-weighted sagittal sequence on lumbar spine

(b) T2-weighted sagittal sequence on lumbar spine

(c) STIR sagittal sequence on lumbar spine

(d) T1-weighted sagittal sequence on dorsal spine

(e) T2-weighted sagittal sequence on dorsal spine

(f) STIR sagittal sequence on dorsal spine

Non-enhanced TI weighted images $(a, b)$ of the spine were normal. T2 and STIR weighted sequences $(\mathrm{c}-\mathrm{f})$ showed a mild swelling and linear hyperintense signal from D11 level and within the conus medullaris

to D11 level. A therapy with high dose intravenously administered methylprednisone was started $(20 \mathrm{mg} / \mathrm{kg}$ per day).

Tests for HIV, cytomegalovirus, measles, varicella zoster, Treponema pallidum, borreliosis, Epstein - Barr virus, Mycoplasma were all negative. Serum HSV -2 immunoglobulin IgG and IgM were

${ }^{\star}$ Correspondence to: Dott.ssa Michaela Cellina, Dirigente Medico - Radiologia, ASST Fatebenefratelli Sacco, Ospedale. Fatebenefratelli e Oftalmico, Piazza Principessa Clotilde 3, 20121 Milano, Italy; Tel: 0263632424; E-mail: michaela.cellina@asst-fbf-sacco.it

Received: September 10, 2018; Accepted: September 21, 2018; Published: September 26, 2018 
increased (IgG index: 1.27 [normal range: $0-0.9$ ], IgM index: $>3.5$ [normal range: 0-0.9]).

White blood cell count was within the normal range $\left(6.4^{\star} 10^{3} / \mathrm{ul}\right.$ [normal range: $4-11^{\star} 10^{3} / \mathrm{ul}$ ], Hemoglobin was slightly reduced $(10.7$ $\mathrm{g} / \mathrm{dL}$ [normal range: $12-16 \mathrm{~g} / \mathrm{dL}$ ], $\mathrm{C}$ reactive protein $(0.75 \mathrm{mg} / \mathrm{dL}$ [normal range: $0-5 \mathrm{mg} / \mathrm{dL}$ ], and the other laboratory tests were within the normal limits. Lumbar puncture (LP) was therefore performed and CSF analysis showed increased albumin ( $49 \mathrm{mg} / \mathrm{dL}$ ), lymphocytic pleocytosis $(158 / \mu \mathrm{L})$, and normal glucose level $(60 \mathrm{mg} / \mathrm{dL})$. HSV-2 polymerase chain reaction in CSF was positive ( 45 copies/ $\mu \mathrm{L}$ ); CSF aerobic and fungal cultures, Enterovirus RT-PCR, West Nile virus IgM and IgG, VDRL, oligoclonal bands were negative.

Parenteral acyclovir ( $250 \mathrm{mg} 4$ times a day) was started. The patients showed a worsening of her status, with areflexia and paraparesis and flaccid paralysis involving both legs; an inconstant adduction movement of the thighs (left > right) was visible. Moreover, she reported a sensation of heat in the lower left limb and progressive constipation. A slight increase of $\mathrm{C}$ reactive protein $(8 \mathrm{mg} / \mathrm{dL})$ was observed.

A follow up contrast-enhanced MRI (10 days after the first one, Figure 2) was performed and showed an increase in abnormal signal intensity of conus medullaris and dorsal cord edema with associated diffuse intramedullary enhancement and sacral roots enhancement. Antiviral therapy was increased to Acyclovir $750 \mathrm{mg} 3$ times/day plus Methylprednisolone $1 \mathrm{~g} /$ day for 10 days. Gradual improvement was observed 15 days after the increase of the antiviral treatment.

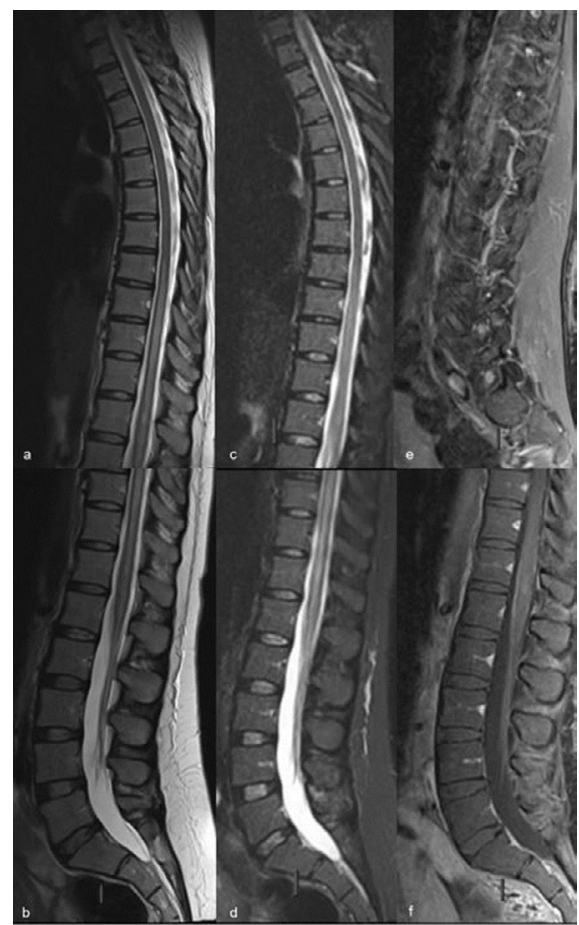

Figure 2. Follow up enhanced dorsal lumbar spine MRI

(a) T2-weighted sagittal sequence on dorsal spine

(b) T2-weighted sagittal sequence on lumbar spine

(c) STIR sagittal sequence on dorsal spine

(d) STIR sagittal sequence on dorsal spine

(e) T1-weighted with contrast injection sagittal sequence on lumbar spine

(f) T1-weighted with contrast injection sagittal sequence on lumbar spine

Worsening of the MRI findings, with signal alterations in T2 and STIR weighted images (a - d) up to D12 level. T1-weighted images with contrast injection showed myeloradiculitis, with enhancement of conus medullaris, both the anteror meninges and the roots of the cauda equine $(e, f)$
Slow general improvement of the patient's neurological functions was observed; however, movement impairment of the right foot was still present at 3 months follow-up.

\section{Discussion}

Several viruses can be causal agents of transverse myelitis, most commonly cytomegalivirus, HSV type 1 or 2, Epstein Barr virus, varicella-zoster virus [1-4]. Although these neurotropic viruses are often suspected of being associated with spinal cord injuries, an early diagnosis of etiology is often difficult, and sometimes confirmed only at postmortem examination. In case of HSV related myelitis, a previous anogenital rash or enlarged inguinal lymph nodes can help a diagnosis; the positivity of serum IgM HSV antibodies can manifest in primary infections but also in cases of viral reactivation [4,5].

HSV 2 can affect all ages but especially age range from 10 to 19 years old and 30 to 39 years old age groups. Immunodeficiency represents a predisposing factor for HSV 2 - myelitis, but also immunocompetent patient can be affected [6].

The most accredited hypothesis is for the pathogenesis of HSV myelitis is that HSV 2 lies dormant in the dorsal sacral root ganglia and, when it reactivates and start a new replication $[4,12]$, the virus can invade the spinal cord forming of necrotizing lesions and a contiguous spread via sacral ganglia to the cervico-dorsal spinal cord, causing ascending myelitis [8-11].

HSV 2 myelitis can present with sensory-motor disturbances of the lower limbs, urinary retention, constipation, and pain in the lumbar zone or in the anogenital region, loss of sensation or paresthesias in various combination [13]. The conus medullaris and lower dorsal spine are predominantly interested, while the ascending form is rare and severe and can manifest with encephalitis, quadriplegia or respiratory muscle paralysis and can have a fatal outcome, especially in immunocompromised patient, with evidence of necrotic and hemorrhagic changes [2,7]. Conditions like HIV infection, tumors [14], diabetes seem to predispose to the ascending diffusion and necrotic evolution of myelitis; even if fatal cases of HSV2 myelitis has been described also in immunocompetent patients.

A recurrence of myelitis symptoms can be observed in about $30 \%$ of patient during the first year after the first presentation [7].

Imaging can help the diagnosis: magnetic resonance imaging may reveal signs of demyelination with or without necrosis, but it is not specific for the different types of neurotropic viruses, therefore lumbar puncture should be performed [15].

In Literature, few cases of PCR- confirmed HSV 2 myelitis in immunocompetent patient are described $[2,3,8,16,17]$ and rare cases describing the MRI appearance of the infection [2,8,9]. The case described by Eberhardt et al. [2] was of a 40-year-old woman who presented with sensory loss with a lower sacral dermatome distribution and urinary retention; at MRI an enlargement with contrast enhancement of sacral radicular fibers was observed, but no signs of involvement of the conus medullaris.

Nardone et al. [16] reported the case of a 60-year-old woman who developed an ascending flaccid tetraparesis, a C6 sensory level, sphincter dysfunction and acute back pain, with a rapid evolution; her MRI showed an involvement of the anterior horn extending from the second cervical to the second dorsal vertebral level with hyperintensity in T2-weighted sequence, follow by a medullar atrophy at the imaging follow up. 
Ellie et al. [17] observed a 76-year-old-woman with HSV ascending myeloradiculitis, who presented with flaccid paraplegia with a D 10 sensory level, urinary retention, fever, anorexia, weight loss and back pain; her MRI demonstrated a linear hyperintense signal in T2weighted images at D 10 level and within the conus medullaris and an enhancement at T1-weighted post contrast medium injection sequence of the posterior meninges and the roots of the cauda.

In our patient, MRI clearly showed myeloradiculitis on T2weighted and gadolinium enhanced T1-weighted sagittal sequences. Although well correlated with the clinical features, MRI findings lacked specificity; PCR assay of CSF is needed for early diagnosis of herpetic central nervous system disorders. In the past, HSV-2 myelitis has been reported as a form of fatal necrotizing myelopathy; however, with PCR and MRI examination, mild surviving cases are increasingly likely to be identified to be correctly managed.

\section{Conflicts of interest}

Authors declare no conflicts of interest.

\section{References}

1. Jacob A, Weinshenker BG (2008) An approach to the diagnosis of acute transverse myelitis. Semin Neurol 28: 105-120. [Crossref]

2. Eberhardt O, Küker W, Dichgans J, Weller M (2004) HSV-2 sacral radiculitis (Elsberg syndrome). Neurology 63: 758-759. [Crossref]

3. Oates JK, Greenhouse PR (1978) Retention of urine in anogenital herpetic infection. Lancet 1: 691-692. [Crossref]

4. Lee CC, Wu JC, Huang WC, Shih YH, Cheng H (2010) Herpes zoster cervical myelitis in a young adult. J Chin Med Assoc 73: 605-610. [Crossref]

5. Figueroa D, Isache C, Sands M, Guzman N (2016) An unusual case of acute transverse myelitis caused by HSV-1 infection. IDCases 5: 29-31. [Crossref]
6. Galanakis E, Bikouvarakis S, Mamoulakis D, et al. (2001) Transverse myelitis associated with herpes simplex virus infection. $J$ Child Neurol 16:866-867.

7. Sarioglu B, Kose SS, Saritas S, et al. (2014) Severe acute disseminated encephalomyelitis with clinical findings of transverse myelitis after herpes simplex virus infection. JChild Neurol 29:1519-1523.

8. Wiley CA, VanPatten PD, Carpenter PM, Powell HC, Thal LJ (1987) Acute ascending necrotizing myelopathy caused by herpes simplex virus type 2. Neurology 37: 17911794. [Crossref]

9. Folpe A, Lapham LW, Smith HC (1994) Herpes simplex myelitis as a cause of acute necrotizing myelitis syndrome. Neurology 44: 1955-1957. [Crossref]

10. Shyu WC, Lin JC, Chang BC, Harn HJ, Lee CC, et al. (1993) Recurrent ascending myelitis: an unusual presentation of herpes simplex virus type 1 infection. Ann Neurol 34: 625-627. [Crossref]

11. Iwamasa T, Yoshitake H, Sakuda H, Kamada Y, Miyazato M, et al. (1991) Acute ascending necrotizing myelitis in Okinawa caused by herpes simplex virus type 2 Virchows Arch A Pathol Anat Histopathol 418: 71-75. [Crossref]

12. Gobbi C, Tosi C, Städler C, Merenda C, Bernasconi E (2001) Recurrent myelitis associated with herpes simplex virus type 2. Eur Neurol 46: 215-218. [Crossref]

13. Nakajima H, Shoji H (2011) Herpes simplex myelitis: differences in clinical manifestations between herpes simplex virus type 1 and type 2. Pathog Enceph 153168.

14. Iwamasa T, Utsumi Y, Sakuda H, Yoshitake H, Kakazu T, et al. (1989) Two cases of necrotizing myelopathy associated with malignancy caused by herpes simplex virus type 2. Acta Neuropathol 78: 252-257. [Crossref]

15. Nakajima H, Furutama D (1998) Herpes simplex virus myelitis: diagnosis by the polymerase chain reaction method. Eur Neurol 39:163-167.

16. Nardone R, Versace V, Brigo F, Tezzon F, et al. (2017) Herpes Simplex Virus Type 2 Myelitis: Case Report and Review of the Literature. Front Neurol 8: 199. [Crossref]

17. Ellie E, Rozenberg F, et al. (1994) Herpes simplex virus type 2 ascending myeloradiculitis: MRI findings and rapid diagnosis by the polymerase chain method. $J$ Neurol Neurosurg Psychiatry 57: 869-870.

Copyright: (C2018 Cellina M. This is an open-access article distributed under the terms of the Creative Commons Attribution License, which permits unrestricted use, distribution, and reproduction in any medium, provided the original author and source are credited. 